University of Nebraska - Lincoln

DigitalCommons@University of Nebraska - Lincoln

Roger Kirby Publications

Research Papers in Physics and Astronomy

April 1968

Far Infrared Properties of Lattice Resonant Modes. I. Isotope Shifts

Roger D. Kirby

University of Nebraska-Lincoln, rkirby1@unl.edu

I.G. Nolt

University of Nebraska - Lincoln

R.W. Alexander Jr.

Cornell University, Ithaca, New York

A.J. Sievers

Cornell University, Ithaca, New York

Follow this and additional works at: https://digitalcommons.unl.edu/physics_kirby

Part of the Physics Commons

Kirby, Roger D.; Nolt, I.G.; Alexander, R.W. Jr.; and Sievers, A.J., "Far Infrared Properties of Lattice Resonant Modes. I. Isotope Shifts" (1968). Roger Kirby Publications. 42.

https://digitalcommons.unl.edu/physics_kirby/42

This Article is brought to you for free and open access by the Research Papers in Physics and Astronomy at DigitalCommons@University of Nebraska - Lincoln. It has been accepted for inclusion in Roger Kirby Publications by an authorized administrator of DigitalCommons@University of Nebraska - Lincoln. 


\title{
Far Infrared Properties of Lattice Resonant Modes. I. Isotope Shifts*
}

\author{
R. D. Kirby, I. G. Nolt, $\dagger$ R. W. Alexander, JR., and A. J. Sievers \\ Laboratory of Atomic and Solid State Physics, Cornell University, Ithaca, New York
}

(Received 20 October 1967)

\begin{abstract}
The frequency shift of a lattice resonant mode associated with the isotopic substitution of an impurity ion has been measured for $\mathrm{NaCl}: \mathrm{Cu}^{+}, \mathrm{KI}: \mathrm{Ag}^{+}$, and $\mathrm{KBr}: \mathrm{Li}^{+}$. For all three systems, the experimental frequency shifts can be described satisfactorily by an Einstein oscillator representation for the resonant mode with the oscillator mass set equal to the impurity-ion mass. The model of Benedek and Nardelli and also Klein does not account for the experimental results of $\mathrm{NaCl}: \mathrm{Cu}^{+}$or $\mathrm{KBr}: \mathrm{Li}^{+}$. The inclusion of anharmonic forces does improve the agreement between theory and experiment, but a large discrepancy still exists for $\mathrm{KBr}: \mathrm{Li}^{+}$.
\end{abstract}

\section{INTRODUCTION}

A LTHOUGH the isotope frequency shift associated with a lattice resonant mode ${ }^{1}$ has been known for some time, the experimental results are not yet really understood. To account for the frequency shift in ${ }^{6} \mathrm{Li}^{+}$- and ${ }^{7} \mathrm{Li}^{+}$-doped $\mathrm{KBr}$, Sievers and Takeno ${ }^{1}$ used a simple lattice model in which long-range forces were neglected. Recently, calculations of the isotope shift have been attempted by Benedek and Nardelli, ${ }^{2}$ using Hardy's deformation dipole model, and by Klein, ${ }^{3}$ using the shell model to describe the perfect lattice. In both of these calculations long-range forces are included only in the description of the perfect lattice. The impurity is still coupled to the lattice with short-range forces. With these models a much smaller isotope shift has been calculated than was found previously either with simple lattice models ${ }^{1,4}$ or by experiment. If the agreement of the simple model with experiment is fortuitous, then the resonant mode must be associated with a very anharmonic potential to account for the discrepancy between theory and experiment. However, because the $\mathrm{Li}^{+}$ion has a small radius and also a small mass, the resonant mode associated with this particular impurity may be anomalous in nature and not a characteristic feature of the coupled system. We have tested this possibility by studying the isotope shifts for the alkali halides $\mathrm{NaCl}$ and $\mathrm{KI}$ containing the heavy $\mathrm{Cu}^{+}$and $\mathrm{Ag}^{+}$impurity ions, respectively. Improved data for the $\mathrm{KBr}: \mathrm{LiBr}$ system also have been obtained. Although the measured shifts for these three dopantlattice systems differ by more than an order of magnitude, the isotope effect indicates that the three systems

\footnotetext{
* Research mainly supported by the U. S. Atomic Energy Commission under Contract No. AT (30-1)-2391, Technical Report No. NYO-2391-63. Additional support was received from the Advanced Research Projects Agency through the use of space and technical facilities of the Materials Science Center at Cornel University.

$\dagger$ Present address: Institute for Astronomy, University of Hawaii, Honolulu, Haw.

1 A. J. Sievers and S. Takeno, Phys. Rev. 140, A1030 (1965).

${ }^{2}$ G. Benedek and G. F. Nardelli, Phys. Rev. 155, 1004 (1967)

${ }^{3}$ M. V. Klein, in Physics of Color Centers, edited by W. Beall Fowler (Academic Press Inc., New York, 1968), Chap. 7.

${ }^{4}$ L. Genzel, K. F. Renk, and R. Weber, Phys. Status Solidi 12, 639 (1965).
}

are indeed similar. The degree of anharmonicity associated with each resonant mode has not been resolved satisfactorily.

In Sec. II, the experimental shifts and resonant-mode linewidths are reported. Included here is a description of the far infrared absorption associated with the unwanted $\mathrm{OH}^{-}$impurity in $\mathrm{NaCl}$.

In Sec. III, the experimental shifts are compared with the shifts predicted by both the simple model with only short-range forces and also with the model of Benedek and Nardelli and of Klein. Anharmonic forces are then discussed. When a large coupling between the resonant mode and other phonon modes is introduced, some agreement between the models and experiment can be realized if the coupling is assumed to be independent of the impurity mass.

\section{EXPERIMENTAL RESULTS}

\section{A. Copper-Ion Impurity}

Far infrared transmission spectra of the doped alkalihalide crystals were measured with a Strong-type lamellar interferometer. ${ }^{5,6}$ The radiation was detected with a liquid-helium-cooled germanium bolometer. By comparing the transmission of the doped samples with the transmission of the pure crystal, the impurityinduced absorption coefficients were obtained. The same crystal-growing techniques were used to obtain all single crystals, i.e., the Kryopolous technique with an argon atmosphere.

The $\mathrm{NaCl}: \mathrm{CuCl}$ transmission measurements were made with the samples at $4.2^{\circ} \mathrm{K}$ and an instrumental resolution of $0.09 \mathrm{~cm}^{-1}$. This resolution is about a factor of 3 better than was available to Weber and Nette in their original investigation of the $\mathrm{NaCl}: \mathrm{CuCl}$ system. ${ }^{7}$ By using isotopically pure ${ }^{63} \mathrm{Cu}^{+}$and ${ }^{65} \mathrm{Cu}^{+}$, the isotope shift has been resolved, and the impurity-induced absorption coefficient for the two isotopes is shown in Fig. 1. The numerical values for the two center frequencies are given in Table I, and the full width at one-

\footnotetext{
${ }^{5}$ P. L. Richards, J. Opt. Soc. Am. 54, 1474 (1964).

6 I. G. Nolt, R. A. Westwig, R. W. Alexander, Jr., and A. J. Sievers, Phys. Rev. 157, 730 (1967).

${ }^{7}$ R. Weber and P. Nette, Phys. Letters 20, 493 (1966).
} 


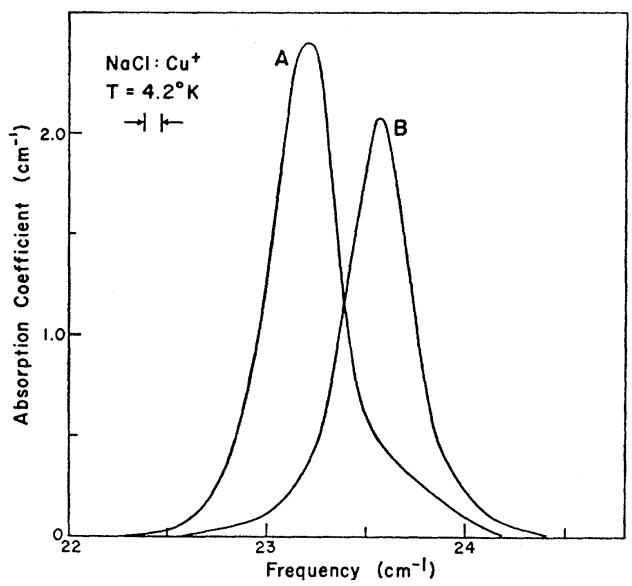

Frg. 1. Impurity-induced absorption coefficient versus frequency for ${ }^{63} \mathrm{Cu}^{+}$and ${ }^{65} \mathrm{Cu}^{+}$impurities in $\mathrm{NaCl}$. Curve A has $1.15 \times 10^{17}$ ${ }^{(65} \mathrm{Cu}^{+}$ions) $/ \mathrm{cm}^{3}$, and curve $\mathrm{B}$ has $1.06 \times 10^{17}\left({ }^{63} \mathrm{Cu}^{+}\right.$ions $) / \mathrm{cm}^{3}$. The interferometer resolution is indicated by the separation of the two arrows.

half-maximum-absorption coefficient $\Gamma$ is given in Table II. Because we have not yet found a consistent method of unfolding the instrumental linewidth from the measured width, only the measured value is given in the table. However, the experimental values of $\Gamma$ probably should be reduced by about $0.02 \mathrm{~cm}^{-1}$ because of the finite instrumental resolution.

A more serious experimental problem has been the identification of unwanted impurities in $\mathrm{NaCl}$. When the natural abundance of $\mathrm{CuCl}$ was added to the melt, an absorption line at $9.2 \mathrm{~cm}^{-1}$ also was observed. Moreover, when isotopic copper in the form of $\mathrm{CuO}$ was added to the melt, a number of additional low-frequency lines appeared. Most of the low-frequency absorptions are due to the $\mathrm{OH}^{-}$impurity. The identification was made by studying $\mathrm{NaCl}$ doped with $\mathrm{OH}^{-}$ions. ${ }^{8}$ The induced absorption in $\mathrm{NaCl}$ for an $\mathrm{OH}^{-}$concen-

TABLE I. Center frequencies for isotopic impurities. A comparison of the experimental resonant-mode frequency ratios with the ratios predicted by the ST model, the BNK model, and with an Einstein oscillator $\left[\left(\Omega \ell / \Omega_{h}\right)=\left(M_{h} / M \ell\right)^{1 / 2}\right]$. Here $\Omega \ell$ and $M \ell$ are the resonant-mode frequency and mass of the lighter impurity, and $\Omega_{h}$ and $M_{h}$ are the frequency and mass of the heavier impurity.

\begin{tabular}{cccccc}
\hline \hline $\begin{array}{c}\text { Resonant- } \\
\text { mode } \\
\text { system }\end{array}$ & $\begin{array}{c}\text { Experimental } \\
\text { resonant } \\
\text { frequency } \\
\left(\mathrm{cm}^{-1}\right)\end{array}$ & $\begin{array}{c}\Omega l / \Omega h \\
\text { (expt.) }\end{array}$ & $\begin{array}{c}\Omega_{l} / \Omega h \\
(\mathrm{ST})\end{array}$ & $\left(M_{h} / M l\right)^{1 / 2}$ & $\begin{array}{c}\Omega l / \Omega h \\
(\mathrm{BNK})\end{array}$ \\
\hline $\mathrm{NaCl}:{ }^{68} \mathrm{Cu}^{+}$ & $23.57 \pm 0.02$ & $1.016 \pm 0.002$ & 1.017 & 1.0158 & $1.013^{\mathrm{a}}$ \\
$\mathrm{NaCl}^{65} \mathrm{Cu}^{+}$ & $23.20 \pm 0.02$ & & & & \\
$\mathrm{KI}: 107 \mathrm{Ag}^{+}$ & $17.37 \pm 0.02$ & $1.008 \pm 0.002$ & 1.0099 & 1.0093 & $1.0065^{\mathrm{b}}$ \\
$\mathrm{KI}: 100 \mathrm{Ag}^{+}$ & $17.23 \pm 0.02$ & & & & \\
$\mathrm{KBr}: 6 \mathrm{Li}^{+}$ & $17.71 \pm 0.05$ & $1.105 \pm 0.004$ & 1.085 & 1.0801 & $1.019 \mathrm{a}, 。$ \\
$\mathrm{KBr}: \mathrm{Li}^{+}$ & $16.07 \pm 0.05$ & & & & \\
\hline
\end{tabular}

a M. V. Klein (Ref. 3).

o Benedek and Nardelli estimated this ratio to be about 1.05 on the basis of their model.

${ }^{8}$ These $\mathrm{NaCl}: \mathrm{OH}^{-}$crystals were kindly supplied by $\mathrm{M}$. V. Kleị and B. Wedding. tration of $60 \mathrm{ppm}^{9}$ is shown in Fig. 2. Not all of this absorption is necessarily due to the $\mathrm{OH}^{-}$impurity itself, as our samples showed an absorption at about $7 \mu,{ }^{9}$ indicating $\mathrm{CO}_{3}-$ impurities. However, the absorption spectrum shown in Fig. 2 does seem to scale roughly with $\mathrm{OH}^{-}$concentration. An oscillator strength ${ }^{10}$ $f=0.02$ has been assigned to the strongest absorption at $9.2 \mathrm{~cm}^{-1}$.

Recently, Bosomworth ${ }^{11}$ has reported an $\mathrm{NaCl}: \mathrm{OH}^{-}$ absorption spectrum which is substantially different from that shown in Fig. 2. He observed a broad peak at $12 \mathrm{~cm}^{-1}$, with a weak absorption at $9.5 \mathrm{~cm}^{-1}$, for an $\mathrm{OH}^{-}$concentration of $1900 \mathrm{ppm}$. The difference between Bosomworth's absorption spectrum and ours probably results from the large difference in $\mathrm{OH}^{-}$concentration. A strong concentration-dependent absorption associated with the internal stretching mode of the $\mathrm{OH}^{-}$ion in the near infrared has been observed by Wedding. ${ }^{12}$

An $\mathrm{NaCl}: \mathrm{OD}^{-}$crystal gave essentially the same spectrum as that shown in Fig. 2, but the $\mathrm{OD}^{-}$stretching mode was not observed by Wedding in the near infrared. ${ }^{9}$ We conclude that the resultant spectrum was associated with the $\mathrm{OH}^{-}$impurity and that the $\mathrm{OD}^{-}$ dopant did not enter the crystal.

Fortunately, the absorption at $22 \mathrm{~cm}^{-1}$ in Fig. 2 is weak. Our $\mathrm{NaCl}: \mathrm{Cu}^{+}$samples contained much less than $60 \mathrm{ppm} \mathrm{OH}^{-}$, and no distortion of the $\mathrm{Cu}^{+}$absorption was observed. As a matter of practical interest, we found that a $\mathrm{Harshaw}^{13} \mathrm{NaCl}$ sample showed a far

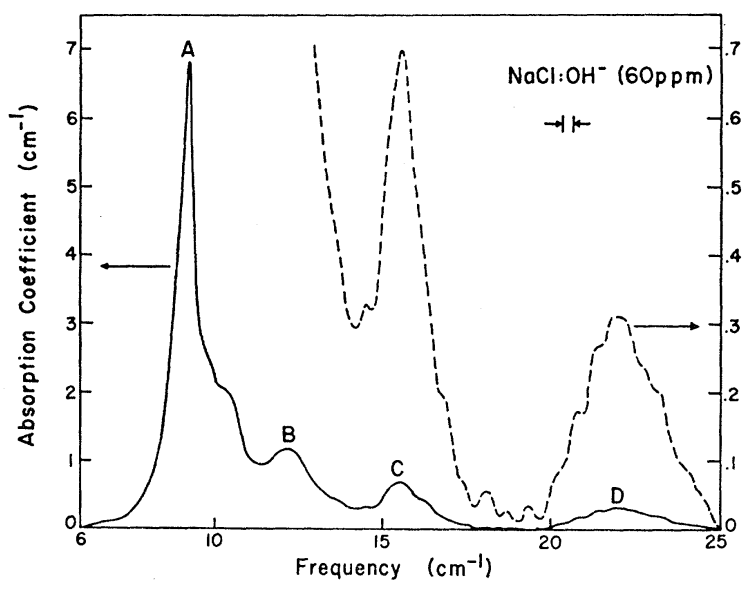

FIG. 2. Impurity-induced absorption coefficient versus frequency for $\mathrm{NaCl}: \mathrm{OH}^{-}$. Note the factor-of- 10 change in the absorption coefficient scale for the dotted curve. The small "wiggles" on the dotted curve are instrumental. Line A is at $9.2 \mathrm{~cm}^{-1}$, line $\mathrm{B}$ is at $12.2 \mathrm{~cm}^{-1}$, line $\mathrm{C}$ is at $15.6 \mathrm{~cm}^{-1}$, and line $\mathrm{D}$ is at $22.0 \mathrm{~cm}^{-1}$. The interferometer resolution is indicated by the separation of the two arrows. The sample temperature was $4.2^{\circ} \mathrm{K}$.

$9 \mathrm{~B}$. Wedding (private communication).

${ }^{10}$ A. J. Sievers, Phys. Rev. Letters 13, 310 (1964).

11 D. R. Bosomworth, Solid State Commun. 5, 681 (1967)

${ }^{12} \mathrm{~B}$. Wedding, Ph.D. thesis, University of Illinois, 1967 (unpublished)

${ }_{13}$ The $\mathrm{NaCl}$ crystal was obtained from the Harshaw Chemical $\mathrm{Co}_{,}$, Cleveland, Ohio. 
infrared spectrum similar to that of Fig. 2, corresponding to a few $\mathrm{ppm} \mathrm{OH}^{-}$.

\section{B. Silver-Ion Impurity}

The KI:AgI system ${ }^{10}$ exhibits an impurity-induced absorption at about $17.3 \mathrm{~cm}^{-1}$. While the relative-mass ratio of the two isotopes $(107 / 109)$ is considerably smaller than for the copper ion, it has been possible to detect the isotopic frequency shift by using isotopically pure ${ }^{107} \mathrm{Ag}^{+}$and ${ }^{109} \mathrm{Ag}^{+}$in the form of $\mathrm{Ag}$ metal as a dopant. ${ }^{14,15}$ Again the far-infrared-transmission measurements were made with the samples at $4.2^{\circ} \mathrm{K}$ and an instrumental resolution of $0.09 \mathrm{~cm}^{-1}$. The impurityinduced absorption coefficients for the two isotopes are shown in Fig. 3. The center frequencies are recorded in Table I, and the full width at one-half-maximum absorption is given in Table II. Note that the linewidth is much smaller than had been estimated previously. ${ }^{10}$

All transmission measurements were made soon after the crystals were grown because this dopant-lattice combination has the curious property of not being stable at room temperature. By monitoring the strength of the far-infrared absorption at low temperature as a function of the length of time that the crystal is at room temperature, a marked decrease in the line strength has been measured over a three-month period. The conversion process can be increased by heating the crystals to $140^{\circ} \mathrm{C}$. At this temperature, the crystal turns a distinct yellow color. Upon cooling to room temperature, the sample returns to a normal clear appearance.

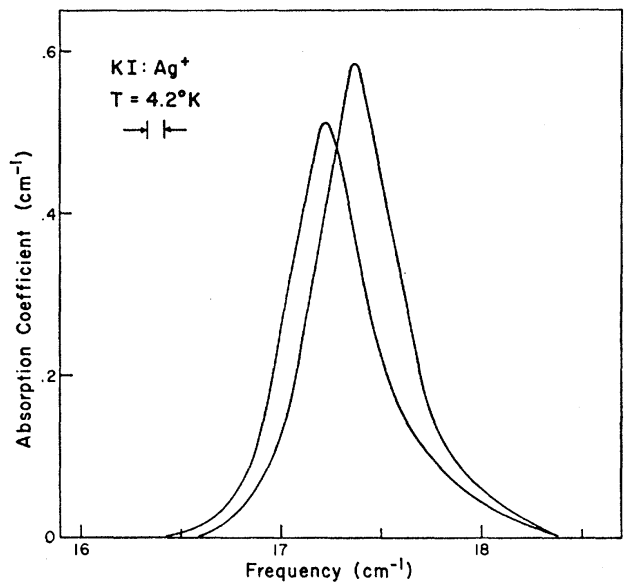

FIG. 3. Impurity-induced absorption coefficient versus frequency for ${ }^{107} \mathrm{Ag}^{+}$and ${ }^{109} \mathrm{Ag}^{+}$impurities in $\mathrm{KI}$. No $\mathrm{Ag}^{+}$concentrations are given because of the uncertainty about what fraction of the $\mathrm{Ag}^{+}$ ions are actually participating in the resonant mode. (See Sec. II B of this paper.) The interferometer resolution is indicated by the separation of the two arrows.

${ }^{14}$ The isotopic copper, silver, and lithium used to dope our samples were obtained from Oak Ridge National Laboratory, Oak Ridge, Tenn.

${ }_{15}$ The crystals were grown by the Materials Science Center Crystal Growing Facility at Cornell University.

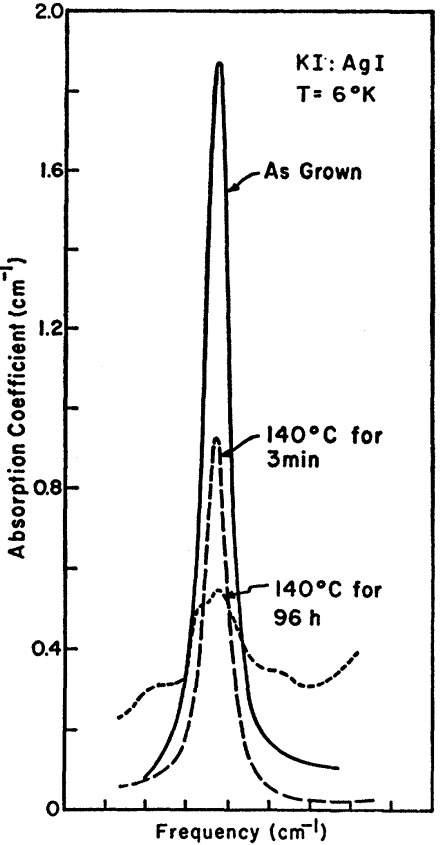

FIG. 4. Temperature instability of the silver-activated resonantmode in KI. The far-infrared transmission was measured with the crystal at $6^{\circ} \mathrm{K}$ between each cycle to $413^{\circ} \mathrm{K}$. For this low-resolution study, the line shape is determined by the instrumental width of $1 \mathrm{~cm}^{-1}$.

When the sample is then cooled to liquid-helium temperatures, the strength of the resonant mode is less than before the heating cycle. The line strength at different stages of the heating cycle is shown in Fig. 4 . As the absorption at $17.3 \mathrm{~cm}^{-1}$ disappears, a new absorption line appears at about $78 \mathrm{~cm}^{-1}$. Although the kinetics of this transformation have not yet been studied in detail, one possible explanation of these results is that $\mathrm{AgI}$ domains may be formed in the crystal at room temperature and above, resulting in a decrease in the resonantmode strength. The 78- $\mathrm{cm}^{-1}$ absorption line is in the appropriate frequency region to be associated with the infrared-active transverse optic mode for the small AgI crystallites.

\section{Lithium-Ion Impurity}

The observation of the ${ }^{6} \mathrm{Li}^{+}$and ${ }^{7} \mathrm{Li}^{+}$resonant-mode frequency shift in $\mathrm{KBr}$ was reported in $1965 .^{1}$ The

TABLE II. Linewidths for isotopic impurities. A comparison of the experimental resonant-mode linewidths with the linewidths predicted by the ST model.

\begin{tabular}{ccc}
\hline $\begin{array}{c}\text { Resonant-mode } \\
\text { system }\end{array}$ & $\begin{array}{c}\Gamma\left(\mathrm{cm}^{-1}\right) \\
\text { Experimental }\end{array}$ & $\begin{array}{c}\Gamma\left(\mathrm{cm}^{-1}\right) \\
(\mathrm{ST})\end{array}$ \\
\hline $\mathrm{NaCl}:{ }^{63} \mathrm{Cu}^{+}$ & $0.38 \pm 0.05$ & 0.57 \\
$\mathrm{NaCl}:{ }^{65} \mathrm{Cu}^{+}$ & $0.40 \pm 0.05$ & 0.56 \\
$\mathrm{KI}: 107 \mathrm{Ag}^{+}$ & $0.47 \pm 0.05$ & 0.72 \\
$\mathrm{KI}:{ }^{109} \mathrm{Ag}^{+}$ & $0.45 \pm 0.05$ & 0.72 \\
$\mathrm{KBr}:{ }^{6} \mathrm{Li}^{+}$ & $0.60 \pm 0.05$ & 0.21 \\
$\mathrm{KBr}:{ }^{7} \mathrm{Li}^{+}$ & $0.43 \pm 0.05$ & 0.15 \\
\hline
\end{tabular}




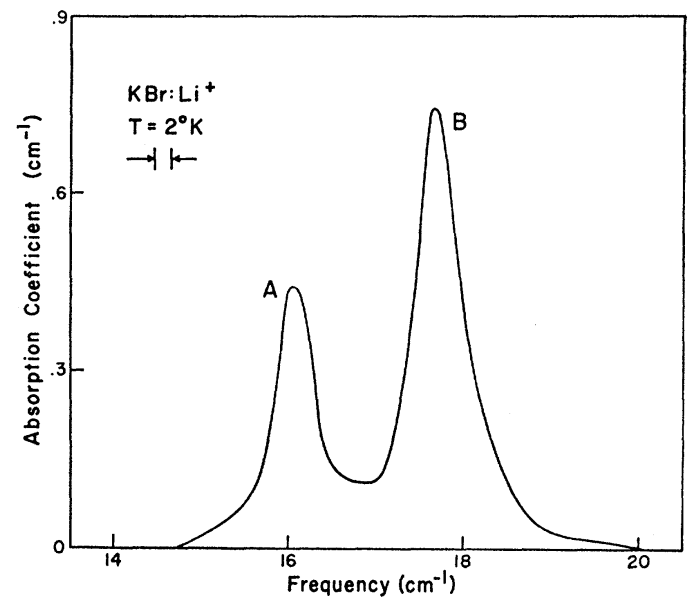

FIG. 5. Impurity-induced absorption coefficient versus frequency for ${ }^{6} \mathrm{Li}^{+}$and ${ }^{7} \mathrm{Li}^{+}$impurities in $\mathrm{KBr}$. Peak $A$ corresponds to $1.9 \times 10^{16}\left({ }^{7} \mathrm{Li}^{+}\right.$ions $) / \mathrm{cm}^{3}$, and peak $B$ corresponds to $3.7 \times 10^{16}$ $\left({ }^{6} \mathrm{Li}^{+}\right.$ions $) / \mathrm{cm}^{3}$. The interferometer resolution is indicated by the separation of the two arrows.

resolution of the grating monochromator was barely sufficient to distinguish the experimental frequency shift from a mass ${ }^{1 / 2}$ dependence. Measurements obtained with the interferometer have increased the precision of both the frequency shift and the linewidths. An impurity-induced absorption spectrum which contains an enriched ${ }^{6} \mathrm{Li}^{+}$and ${ }^{7} \mathrm{Li}^{+}$mixture is shown in Fig. 5. The sample was maintained at $2^{\circ} \mathrm{K}$, and the instrumental resolution was $0.18 \mathrm{~cm}^{-1}$. A careful investigation of these two absorption lines for a variety of lithium concentrations has revealed a concentration effect. By varying the lithium concentration from $2 \times 10^{15} \mathrm{Li}^{+}$ ions $/ \mathrm{cm}^{3}$ to $2.4 \times 10^{18} \mathrm{Li}^{+}$ions $/ \mathrm{cm}^{3}$ (three orders of magnitude), a change has been detected for both the linewidth and the center-frequency position. Heretofore such concentration effects have been overlooked.

The center frequency of the resonance absorption varies linearly with $\mathrm{Li}^{+}$concentration. A frequency shift of $0.07 \mathrm{~cm}^{-1}$ is observed when the concentration is changed from $2 \times 10^{17}$ to $2 \times 10^{15}$ ions $/ \mathrm{cm}^{3}$ for ${ }^{7} \mathrm{Li}^{+}$in $\mathrm{KBr}$. The value of the experimental frequency ratio $\Omega_{6} / \Omega_{7}$ in Table I was obtained from crystals of the lowest concentrations measured. We now estimate the frequency shift due to the concentration dependence of the average lattice constant.

By measuring the frequency shifts of a resonant mode with static stress applied to the crystal, the coupling of the first excited state to long-wavelength phonons can be measured. ${ }^{16}$ The stress perturbation produces a crystal strain which can be resolved into strain components transforming according to the irreducible representations of the octahedral point group. Three coefficients $A, B$, and $C$ prescribe the dependence of the resonant-mode frequency upon the fully symmetric

${ }^{16}$ I. G. Nolt and A. J. Sievers, Phys. Rev. Letters 16, 1103 (1966).
$\left(A_{1 g}\right)$, the tetragonal $\left(E_{g}\right)$, and the trigonal $\left(T_{2 g}\right)$ strain components, respectively. The three coefficients have been determined in Ref. 16 for $\mathrm{KBr}: \mathrm{Li}^{+}$. The coefficient for the strain component which transforms as the $A_{1 g}$ representation exhibits directly the dependence of the resonant-mode frequency upon unit volume change. The transition energy is

$$
E=\hbar \Omega+A(\Delta V / V) \text {. }
$$

We estimate the frequency shift by first calculating the average lattice constant $\vec{a}$ from the known impurity concentration by using Vegard's relation ${ }^{17}$

$$
\bar{a}=a_{1}+\left(a_{2}-a_{1}\right) x,
$$

where $a_{1}$ and $a_{2}$ are the lattice constants of $\mathrm{KBr}$ and $\mathrm{LiBr}$, respectively, and $x$ is the molar concentration of LiBr. For $a_{1}=6.58 \AA, a_{2}=5.50 \AA$, and $2 \times 10^{17} \mathrm{Li}^{+}$ions/ $\mathrm{cm}^{3}$, the calculated frequency shift due to the volume change is $0.006 \mathrm{~cm}^{-1}$. This number is about an order of magnitude smaller than the observed shift. Such a large discrepancy might be resolved if, in addition to the strain interaction, there was a dynamic coupling between $\mathrm{Li}^{+}$ions.

The concentration dependence of the full width at half-maximum absorption is shown in Fig. 6. The data have been corrected for the instrumental width, assuming a Gaussian absorption line and slit function. The slope of the line in Fig. 6 indicates that the linewidth depends quite weakly upon the concentration, varying as the concentration to the 0.1 power approximately. From earlier measurements on the stressinduced shifts of the lithium resonant mode an estimate of the random strain broadening can be made. ${ }^{16}$ For random static stresses on the order of $\frac{1}{2} \mathrm{~kg} / \mathrm{mm}^{2}$ in the crystal, an increased width of about $0.1 \mathrm{~cm}^{-1}$ can be expected. A good estimate of the true resonant-mode width will not be possible until the problem of lattice

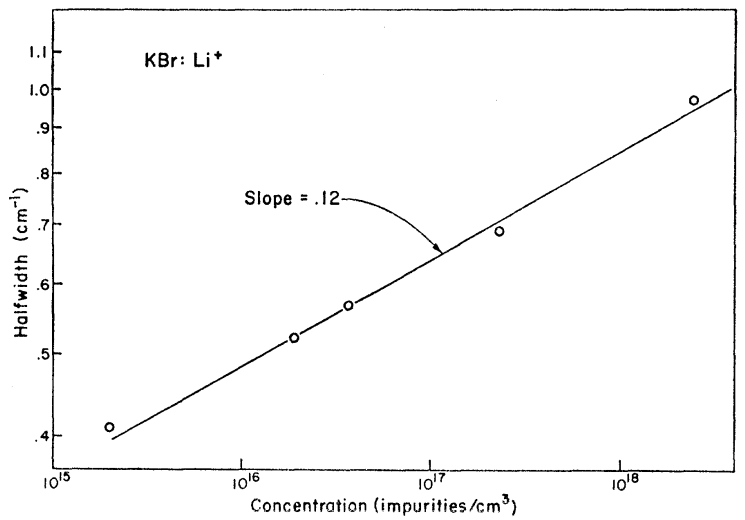

FIG. 6. Concentration dependence of the full width at halfmaximum absorption for $\mathrm{KBr}: \mathrm{Li}^{+}$. The experimental widths have been corrected for the instrumental width, assuming a Gaussian absorption line and slit function. Note the log-log coordinates.

${ }^{17}$ L. Vegard, Z. Physik 5, 17 (1921). 
strains has been studied in detail, but the experimental value of $0.43 \mathrm{~cm}^{-1}$ in Table II is clearly an upper bound.

\section{DISCUSSION OF RESULTS}

\section{A. Isotope Shift in the Harmonic Approximation}

In the model which Sievers and Takeno (hereafter called ST) used for low-frequency resonant modes, only nearest-neighbor forces were considered. The central and noncentral force constants of the host crystal were set equal to each other, while both the impurity mass $M^{\prime}$ and its nearest-neighbor force constant $K^{\prime}$ were taken to be different than those of the host lattice. From their Eq. (3) we can obtain a simple expression for the ratio of the resonant-mode frequencies for two isotopic impurity ions. If the light isotope is labeled $M_{\ell}$ with resonant-mode frequency $\Omega \ell$, and the heavy isotope $M_{h}$ with frequency $\Omega_{h}$, then the ratio of the frequencies is

$$
\frac{\Omega_{l}}{\Omega_{h}}=\left(\frac{M_{h}}{M_{\ell}}\right)^{1 / 2}\left[1-3\left(\frac{M_{h}}{M_{\ell}}-1\right)\left(\frac{\Omega_{h}}{\omega_{D}}\right)^{2}\right]^{-1 / 2},
$$

where $\omega_{D}$ is the Debye frequency for the lattice. Note that Eq. (3) predicts that the frequency ratio is always larger than that obtained with an Einstein oscillator, namely, larger than $\left(M_{h} / M_{\ell}\right)^{1 / 2}$.

The values for $\omega_{D}$ were taken to be $164 \mathrm{~cm}^{-1}$ for $\mathrm{NaCl}, 101 \mathrm{~cm}^{-1}$ for KI, and $121 \mathrm{~cm}^{-1}$ for $\mathrm{KBr}$, and the ratios are compared to the experimental results in Table I.

Our experimental results may also be compared with the predictions of the model containing longrange Coulomb interactions which was formulated by Benedek and Nardelli. ${ }^{2}$ Here a monovalent impurity with a different mass and nearest-neighbor central force constant is substituted for a host ion. Using Hardy's deformation dipole model to obtain the perfectlattice-phonon spectrum, Benedek and Nardelli (hereafter called $\mathrm{BN}$ ) have estimated the frequency ratio for isotopic $\mathrm{Li}^{+}$in $\mathrm{KBr}$. Also, Benedek has made a similar computer calculation for $\mathrm{Ag}^{+}$in $\mathrm{KI}^{18}$ This result is given in Table I.

Starting with the BN model, Klein has derived an expression for the resonant-mode frequency of a positive impurity for the limit of low frequencies. If the same force constants are assumed for both isotopic impurities, then the frequency ratio of the two resonant modes is ${ }^{3}$

$$
\frac{\Omega_{\ell}}{\Omega_{h}}=\left[\frac{M_{h}+\delta M_{+}}{M_{\ell}+\delta M_{+}}\right]^{1 / 2},
$$

where $\delta M_{+}$is an effective mass representing a hostlattice contribution to the impurity mass. Since $\delta M_{+}>0$, this frequency ratio is always less than the Einstein oscillator value, in contrast with the ST model de-

${ }^{18} \mathrm{G}$. Benedek (private communication). scribed earlier. Klein has calculated the resonant-mode parameters, using the $\mathrm{BN}$ model, but with the perfectlattice spectrum obtained from the shell model. $\mathrm{He}$ finds $\delta M_{+}=15.72 \mathrm{amu}$ for $\mathrm{Cu}^{+}$in $\mathrm{NaCl}$ and $\delta M_{+}=20.4$ amu for $\mathrm{Li}^{+}$in $\mathrm{KBr}$. The calculated frequency ratio is compared with the experimental numbers in Table I.

We see that the ST model is in somewhat better agreement with the experimental results than the BN model. The ST model gives the correct isotope shift (within the experimental error) for $\mathrm{NaCl}: \mathrm{Cu}^{+}$and $\mathrm{KI}: \mathrm{Ag}^{+}$, and gives about $80 \%$ of the experimental shift for $\mathrm{KBr}: \mathrm{Li}^{+}$. The $\mathrm{BN}$ model agrees with experiment for $\mathrm{KI}: \mathrm{Ag}^{+}$, gives about $80 \%$ of the experimental shift for $\mathrm{NaCl}: \mathrm{Cu}^{+}$, and only about $20 \%$ for $\mathrm{KBr}: \mathrm{Li}^{+}$.

\section{B. Anharmonic Effects}

Clearly, a model such as that of Benedek and Nardelli and Klein (BNK), which includes both long-range Coulomb forces as well as short-range repulsive forces, should be a good approximation to the actual impuritylattice system. We now look for anharmonic contributions to the resonant-mode frequency which could influence the isotope shift.

Two different anharmonic effects are described here. One contribution to the isotope shift can arise from the anharmonic nature of the potential which describes the resonant-mode oscillator. Another contribution can arise from the modulation of this potential by other lattice modes. To be more specific, we describe the resonant mode by an oscillator coordinate $Q$; then, in the harmonic approximation, the Hamiltonian for the mode is

$$
H=(1 / 2 M) P^{2}+\frac{1}{2} M \Omega^{2} Q^{2},
$$

where $M$ is the effective mass of the oscillator, and $\Omega$ is the oscillator frequency. A perturbation which does not depend on the other lattice-mode coordinates (we label this a static perturbation) is

$$
H^{\prime}=C Q^{4},
$$

where $C$ is a constant. This static effect will be contrasted with the dynamic perturbation which shall be treated shortly. By first-order perturbation theory the 0-1 transition energy for the anharmonic oscillator is

$$
E=\hbar \Omega+\epsilon / M,
$$

where $\epsilon=3 C h^{2} / M \Omega^{2}$ is independent of the oscillator mass $M$. Therefore the anharmonic contribution to the transition energy is proportional to $1 / M$. From the known values of the resonant-mode frequencies in Table I, $\epsilon$ can be calculated. For the ST model, the oscillator mass is the impurity-ion mass. Inserting these masses in Eq. (7), we find that the anharmonic contribution to the resonant-mode frequency is about $37 \%$ of the harmonic contribution for $\mathrm{KBr}:{ }^{6} \mathrm{Li}^{+}$. In other words, large anharmonic effects are necessary to explain 
the isotope shift on the basis of the ST model. For larger mass-impurity ions, the anharmonic contribution to the isotope shift becomes smaller. In fact, if we assume that the coefficient $\epsilon$ in Eq. (7) determined for $\mathrm{Li}^{+}$in $\mathrm{KBr}$ also roughly describes the anharmonic contribution for $\mathrm{Cu}^{+}$in $\mathrm{NaCl}$, then the calculated frequency ratio becomes

$$
\Omega(63) / \Omega(65)=1.018 \text {. }
$$

This value is still within the experimental error of the measured value in Table $\mathrm{I}$.

The BNK model requires a much larger anharmonic coefficient $\epsilon$ because of the large effective mass in Eq. (7). In fact, as we now show, even a very anharmonic potential such as a square well cannot remove the disagreement with experiment. For the square well the transition energy varies as $1 / M$, rather than $1 / \sqrt{ } M$ as for the harmonic oscillator. Klein ${ }^{3}$ found $\Omega(6) / \Omega(7)$ $=1.02$ for $\mathrm{Li}^{+}$in $\mathrm{KBr}$ in the harmonic approximation. Then, for the square-well potential, this ratio should only change to $\Omega(6) / \Omega(7) \approx 1.04$, which is only $40 \%$ of the experimental shift. By itself, then, this static perturbation cannot remove the discrepancy.

Another effect can arise from the dynamical motion of the lattice, and we now consider this possibility. To illustrate this problem, the perturbation to the harmonic-oscillator resonant mode is now

$$
H^{\prime}=\frac{1}{2} Q^{2} \frac{1}{\sqrt{ } N} \sum_{k} b_{k} q_{k},
$$

where $Q$ is again the coordinate of the resonant mode, and $q_{k}$ is the coordinate of the $k$ th lattice mode. (Later, we shall approximate these lattice modes by phonon modes of the unperturbed lattice.) The anharmonic coupling coefficient of this mode to the resonant mode is $b_{k}$, and $N$ is the number of degrees of freedom of the lattice. Because of the linear coupling, the transition energy is

$$
E=\hbar \Omega+\frac{1}{2 M \Omega} \frac{1}{\sqrt{ } N} \sum_{k} b_{k} q_{k} .
$$

We shall now estimate the magnitude of this effect for $\mathrm{Li}^{+}$in $\mathrm{KBr}$ by using the static-stress results discussed earlier. The dependence of the resonant-mode frequency upon a unit-volume change of the lattice has already been given by Eq. (1). We assume that the modes in the perturbed lattice can be represented by the unperturbed phonons of the perfect crystal. Noting that the local strain for long-wavelength plane waves of wave vector $k$ varies as $k q_{k},{ }^{19}$ we set $b_{k}=b k$ in Eq. (9).

${ }^{19}$ R. H. Silsbee, Phys. Rev. 128, 1726 (1962); R. H. Silsbee, in Proceedings of the Third International Congress on Quantum Electronics, edited by P. Grivet and N. Bloembergen (Columbia University Press, New York, 1964), p. 774; R. H. Silsbee and D. B. Fitchen, Rev. Mod. Phys. 36, 423 (1964); R. H. Silsbee, in Lectures on Optical Properties of Solids, NATO Advanced Study Institute, Freiburg, Germany, 1966, edited by S. S. Mitra (to be published).
The lattice dilation is then ${ }^{20}$

$$
\frac{\Delta V}{V}=\frac{1}{N} \sum_{k} k q_{k},
$$

and from Eq. (1)

$$
A=\hbar b / 2 M \Omega \text {. }
$$

Putting this value for $b$ back into Eq. (9), the meansquare breadth associated with the transition is

$$
\left\langle(E-\hbar \Omega)^{2}\right\rangle=\Delta^{2}>\frac{A^{2}}{\mathrm{~N}} \sum_{k} k^{2}\left\langle q_{k}^{2}\right\rangle .
$$

The inequality sign is used because the tetragonal and trigonal contributions have been neglected. At $0^{\circ} \mathrm{K}$

$$
\left\langle q_{k}{ }^{2}\right\rangle=\hbar / 2 m \omega_{k} .
$$

Thus, using the Debye approximation, we find for the second moment

$$
\Delta^{2}>\frac{A^{2} \hbar}{2 m v^{2}} \int_{0}^{\omega \mathrm{D}} \frac{d \omega}{\omega^{3}} \frac{\omega_{D^{3}}}{\omega^{2}}
$$

where $k=\omega / v$, and $v$ is the velocity of sound. Also, $h \omega_{D}=k \theta_{D}$, where $\theta_{D}$ is the Debye temperature, and $\omega_{D}$ is the cutoff frequency. For this case

$$
\Delta>\frac{1}{2} A\left(h \omega_{D} / 2 m v^{2}\right)^{1 / 2} .
$$

If we assume that $m$ is the acoustic mass, i.e.,

$$
m=\frac{1}{2}\left[m\left(\mathrm{~K}^{+}\right)+m\left(\mathrm{Br}^{-}\right)\right],
$$

and take the measured value of $A=830 \mathrm{~cm}^{-1}$, then $\left(h \omega_{D} / 2 m v^{2}\right)^{1 / 2}=0.057$, and

$$
\Delta>24 \mathrm{~cm}^{-1}
$$

for $\mathrm{Li}^{+}$in $\mathrm{KBr}$. From Table II the measured width of the resonant mode is at least a factor of 60 smaller than the value of $\Delta$ above. Thus the square root of the second moment is not directly related to the linewidth of the resonant mode. Because of the large linear coupling of the resonant mode to long-wavelength phonons, the modulation of the resonant-mode energy levels does introduce characteristic properties into the absorption spectrum. The second moment which we have estimated above is to be associated with the breadth of the resonant mode plus phonon sideband. In fact, modulation which is linear in the phonon coordinates does not contribute to the width of the resonant mode in first order. ${ }^{19}$ The sharp zero-phonon lines which have been studied in the optical spectrum are a much better known example of this motional effect of the lattice. ${ }^{19,21}$ There has been some confusion

\footnotetext{
${ }^{20}$ C. Kittel, Quantum Theory of Solids (John Wiley \& Sons, Inc., New York, 1963), p. 23.

${ }_{21}$ T. Timusk and M. V. Klein, Phys. Rev. 141, 664 (1966).
} 
about the credibility of identifying low-frequency transitions with zero-phonon lines because the adiabatic approximation is usually used in the optical problem. However, a number of investigators ${ }^{3,19,22}$ have indicated that if the phonon-impurity interaction shifts but does not mix the impurity states, then the adiabatic approximation is not required.

Because of the strong coupling between the resonant mode and the phonons, the zero-phonon resonant-mode transition $E^{\circ}$ will be shifted from the value given by a harmonic oscillator. Linear coupling to the phonon field will give a shift in second order, while quadratic coupling produces a shift in first order. As we are mainly interested in the magnitude of this shift, we continue with our study of the linear coupling. In second order the center frequency $E^{\circ}$ is given by

$$
E^{\circ}=\hbar \Omega-\frac{A^{2}}{2 m N} \sum_{k} \frac{k^{2}}{\omega_{k}^{2}} .
$$

In the Debye approximation

$$
E^{\circ}=\hbar \Omega-\frac{1}{3} A\left(A / 2 m v^{2}\right),
$$

and for ${ }^{7} \mathrm{Li}^{+}$the correction term becomes

$$
\Delta E^{\circ}=\frac{1}{3} A\left(A / 2 m v^{2}\right)=6.2 \mathrm{~cm}^{-1} .
$$

As only the hydrostatic coupling term has been used, this $\Delta E^{\circ}$ probably represents a lower bound. For both the ST model and also the BNK model, $\Delta E^{\circ}$ will only reduce the calculated isotope shift from that given by the harmonic approximation. That is, the correction we have found is in the wrong direction. The reduction occurs because the coupling constant $A^{2}$ varies inversely as the oscillator mass itself [see Eq. (11)].

A second-order dipole-moment coupling of the resonant mode to the lattice phonons could produce a coupling term which would not depend on the impurity mass; hence the correction would then be in the right direction. Such a coupling would increase the isotope shift over that given by a harmonic oscillator. No evidence for such a coupling mechanism has as yet been found, but perhaps accurate stress measurements on the ${ }^{6} \mathrm{Li}^{+}$and ${ }^{7} \mathrm{Li}^{+}$resonant modes will demonstrate whether or not the linear coupling coefficient does indeed depend on the impurity mass.

In order to better understand some of the other contributions to the isotope effect, the temperaturedependent properties of these resonant modes are now being measured. Apparently, only dynamic perturbations such as the one considered above can lead to agreement between the BNK model and the large experimental isotope shifts observed for $\mathrm{Li}^{+}$and $\mathrm{Cu}^{+}$. For $\mathrm{Ag}^{+}$the experimental errors for the frequency

\footnotetext{
${ }^{22}$ D. E. McCumber, Phys. Rev. 133, A163 (1964).
}

shifts are still large enough to mask any detailed comparison with theory.

\section{SUMMARY}

For both $\mathrm{KBr}: \mathrm{Li}^{+}$and $\mathrm{NaCl}: \mathrm{Cu}^{+}$the resonant-mode isotope shifts are at least as large as given by an Einstein oscillator, with the mass of the oscillator given by the impurity ion mass itself. This experimental result has been contrasted with the BNK model which predicts isotope shifts which are always smaller than those given by the Einstein oscillator. The introduction of anharmonic forces has not improved significantly the agreement between theory and experiment. We have shown that dynamic coupling of the resonant mode to the lattice is important, but a satisfactory answer has not been found. Recently, Krumhansl ${ }^{23}$ has pointed out that the disagreement between the $\mathrm{BNK}$ model and experiment may occur for yet a different reason. Consistent solutions do not follow necessarily from a model in which the impurity is coupled to the lattice by short-range forces while the other ions are coupled together by both short-range and long-range forces. To be self-consistent, a model should treat all ions on an equal basis.

In the course of our investigation, a number of additional properties of lattice resonant modes have been uncovered. The resonant-mode widths are found to be much smaller than previously reported. For $\mathrm{KBr}: \mathrm{Li}^{+}$ a concentration dependence of the linewidth and also the resonant-mode frequency has been observed. Also, the resonant mode has been measured for concentrations as low as $2.5 \times 10^{15}$ ( $\mathrm{Li}^{+}$ions) $/ \mathrm{cm}^{3}$. Although the concentration dependence of the resonant modes for $\mathrm{Cu}^{+}$and $\mathrm{Ag}^{+}$has not yet been investigated, similar concentration effects are expected.

Linewidth values must still be regarded with a degree of caution. For although the contribution from concentration broadening and also from the finite resolving power of the interferometer can be estimated, the broadening from inhomogeneous lattice stains has not been studied yet. The true width of the resonant mode is no doubt still smaller than the widths which we have reported here.

\section{ACKNOWLEDGMENTS}

We wish to thank Dr. B. Wedding for sending us several $\mathrm{NaCl}: \mathrm{OH}^{-}$samples which he had analyzed for $\mathrm{OH}^{-}$concentration, and for allowing us to see his nearinfrared data on these samples. We would also like to acknowledge helpful discussions with Dr. G. Benedek, Professor M. V. Klein, Professor J. A. Krumhansl, and Professor R. H. Silsbee.

${ }^{23} \mathrm{~J}$. A. Krumhansl, Comment at the International Conference on Localized Excitations in Solids, Irvine, September 1967. (unpublished). 